\title{
Spectral hole burning on silicon phthalocyanines isolated in an argon matrix
}

\author{
Dieter Braun a , Arnout Ceulemans ${ }^{\text {a }}$, Bernhard Dick ${ }^{b}$, Hideo Konami ${ }^{\mathrm{c}}$ \\ - Laboratory for Quantum Chemistry. Departement Scheikunde, Katholieke Universiteit Leuven, \\ Celestijnenlaan 200F, 3001 Leuven, Belgium \\ ' Institut für Physikalische und Theoretische Chemie, Universität Regensburg, Universitätsstrasse 31, D-93040 Regensburg, Germany \\ 'Institute for Chemical Reaction Science, Tohoku University, 2-1-1, Katahira, Aoba-ku, Sendai 980, Japan
}

Received 25 April 1994

\begin{abstract}
Irradiation of the monomeric phthalocyanine $\mathrm{Hex}_{3} \mathrm{SiO}$ ( $\mathrm{SiPc}_{\text {) }} \mathrm{OSiHex}_{3}$ isolated in an $\mathrm{Ar}$ matrix at $T=13 \mathrm{~K}$ with the $632.8 \mathrm{~nm}$ line of a $\mathrm{He} / \mathrm{Ne}$ laser produces a resonant spectral hole in the vibrational sideband located about $700 \mathrm{~cm}^{-1}$ above the origin of the $\mathrm{Q}$ band. This hole is accompanied by several sideholes in the origin region. These permit a precise determination of several excited-state vibrational frequencies. For dimeric $\mathrm{Hex}_{3} \mathrm{SiO}(\mathrm{SiPc}-\mathrm{O}-\mathrm{SiPc}) \mathrm{OSiHex}_{3}$ in the same matrix the changes in the absorption spectra after irradiation into the $Q$ band reveal an out-of-plane polarization of the bands which are not present in the monomer absorption. This points to substantial charge-resonance contributions to the excitonic coupling between the two SiPc units in dimeric $\mathrm{Hex}_{3} \mathrm{SiO}(\mathrm{SiPc}-\mathrm{O}-\mathrm{SiPc}) \mathrm{OSiHex}_{3}$.
\end{abstract}

\section{Introduction}

Spectral hole burning is widely applied to the study of homogeneous widths of electronic transitions in organic as well as inorganic materials [1-3]. Hole burning also represents a valuable line-narrowing method for obtaining detailed vibrational structures hidden in inhomogeneously broadened absorption spectra [4-7]. Free base porphyrins [8] and phthalocyanines [9] belong to the first compounds on which successful hole-burning experiments could be performed. The mechanism that leads to the appearance of spectral holes in free base compounds is the tautomerism of the central protons. This mechanism is not possible for porphyrins or phthalocyanines with a single atom or ion in their center. However, transient holes could be burned for unsubstituted zinc porphyrin using the long-lived triplet states as popu- lation reservoir [10]. For metal porphyrins with substituents at the carbon skeleton $[4,11]$ or at the metal $[12,13]$ persistent hole burning could be accomplished. Recently, zinc phthalocyanine derivatives were used for hole burning at a temperature as high as $T=80 \mathrm{~K}$ [14].

In this contribution we want to report on holeburning experiments on silicon phthalocyanines (SiPcs). In these compounds a Si(IV) ion replaces the two central protons of the free base phthalocyanine. Oligomeric silicon phthalocyanines represent ideal model systems for the study of one-dimensional exciton coupling [15] since the overlap of the molecular orbitals of the two aromatic rings is weak [16]. This is due to the relatively large distance of 3.3-3.4 $\AA$ between the phthalocyanine rings [17] compared, for example, with lanthanide-bis-phthalocyanine 
sandwich complexes (ring-ring distance about $2.7 \AA$ for $\mathrm{LuPc}_{2}$ [18]).

It is possible to prepare oligomeric molecules with a defined number of SiPc units [19]. This offers the possibility of studying the buildup of an exciton band in a step-by-step procedure. Especially a more detailed knowledge of the vibronic coupling properties of the excited singlet states of dimeric compounds $[20,21]$ may elucidate the role of charge-resonance contributions which have been found for the corresponding triplet states [22].

\section{Experimental}

The monomeric $\mathrm{Hex}_{3} \mathrm{SiO}(\mathrm{SiPc}) \mathrm{OSiHex}_{3}$ and the dimeric $\mathrm{Hex}_{3} \mathrm{SiO}$ (SiPc-O-SiPc)OSiHex ${ }_{3}$ (Hex: $n$ $\mathrm{C}_{6} \mathrm{H}_{13}$, compare Fig. 1) were prepared according to the method of Wheeler et al. [19]. The purity of the products was checked by NMR spectroscopy. The investigated compounds were placed in an oven in a closed-cycle refrigerator cryostat (Leybold ROK 10300 ) to achieve evaporation at a pressure of approximately $2 \times 10^{-6} \mathrm{mbar}$. The samples were heated to about $140^{\circ} \mathrm{C}$ for the monomer and to about $200^{\circ} \mathrm{C}$ for the dimer. Ar films with a thickness of about $0.3 \mathrm{~mm}$ were prepared with the Ar flow $(10-12 \mathrm{mmol} / \mathrm{h})$

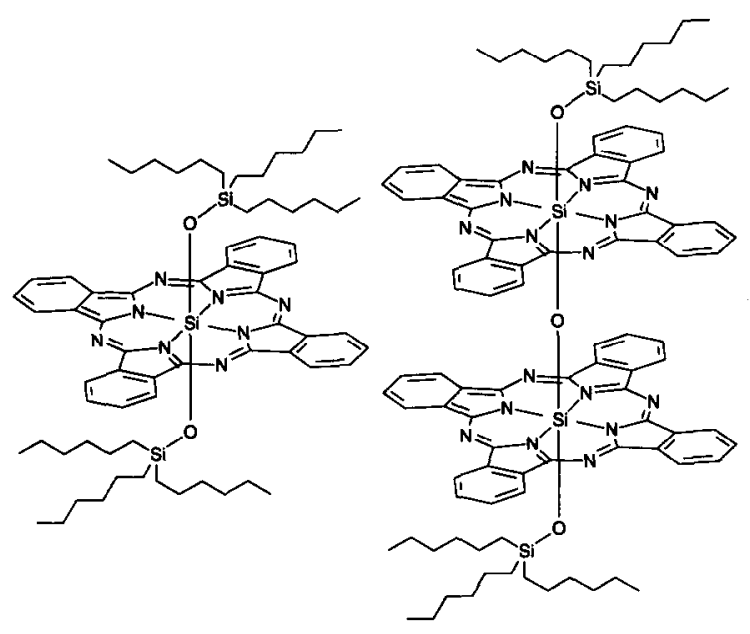

(a)

(b)

Fig. 1. Schematic representation of the SiPc monomer $\mathrm{Hex}_{3} \mathrm{SiO}(\mathrm{SiPc}) \mathrm{OSiHex}_{3}$ (a) and the SiPc dimer Hex 3 SiO (SiPcO-SiPc)OSiHex (b). passing through the oven and guided to a transparent cold surface (LiF substrate) to condense the mixed $\mathrm{Ar} / \mathrm{SiPc}$ gas at a temperature of $T=25 \mathrm{~K}$.

All absorption measurements were performed with a sample temperature of $T=13 \pm 1 \mathrm{~K}$ using a tungsten lamp (Osram Xenophot HLX) and a one-beam procedure. Thus, transmission and reference spectra had to be measured separately. Due to the reflection characteristics of the gratings ( 1200 lines $/ \mathrm{mm}$ ) used in the double monochromator (Spex 1401) the light for the absorption measurements was predominantly polarized in the plane of incidence of the spectrometer. The signals were detected with a cooled photomultiplier and processed by a photon-counting system connected to a computer.

For hole burning the sample was irradiated with the $632.8 \mathrm{~nm}$ line of a He/Ne laser (Spectra Physics $124 \mathrm{~A}$, nominal output power $35 \mathrm{~mW}$ ). Plasma lines were suppressed with an interference filter. The laser beam was expanded to a diameter of about $1.5 \mathrm{~cm}$ with a lens to ensure irradiation of the entire surfaces of the Ar films probed by the absorption measurement. The laser light was guided onto the samples collinear with the beam path for the absorption measurements and was polarized perpendicular to the light for the absorption measurements.

\section{Results and discussion}

\subsection{SiPc monomer}

The lineshape of the Q-band absorption of the monomer in an Ar matrix at low temperature ( $T=13$ $\mathrm{K}$, Fig. 2a) is similar to that observed for solutions in tetrahydrofuran or cyclohexane [15,23]. All bands possess halfwidths of approximately $230 \mathrm{~cm}^{-1}$ and the electronic origin of the $Q$ band appears at 15130 $\mathrm{cm}^{-1}$. It is shifted to the blue by approximately 100 $\mathrm{cm}^{-1}$ relative to the absorption in solutions $[15,23]$. This solvent shift reflects the lower polarizability of the argon matrix.

A rough analysis of the vibrational structure is already possible from the broad-band absorption. Each of the vibrational sidebands displays less than $20 \%$ of the integrated extinction of the origin. This corresponds to Huang-Rhys factors for Franck-Condon modes of $S<0.2$ pointing to similar equilibrium ge- 


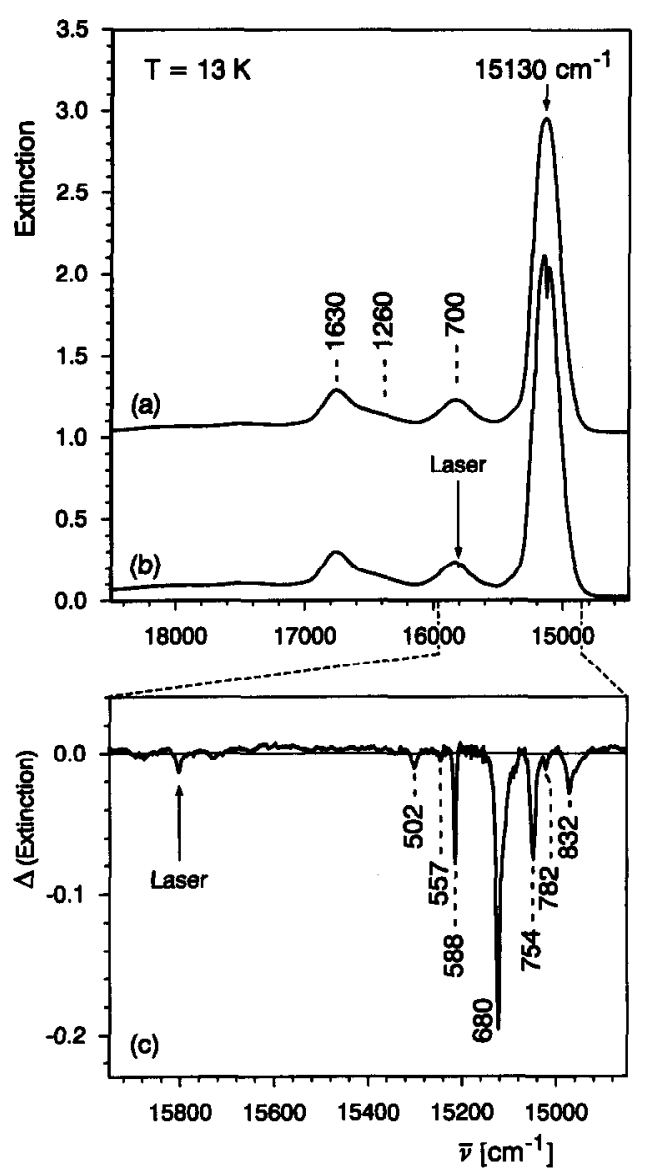

Fig. 2. Absorption spectra of the monomer $\mathrm{Hex}_{3} \mathrm{SiO}(\mathrm{SiPc}) \mathrm{OSiHex}_{3}$ in an Ar matrix at $T=13 \mathrm{~K}$, (a) before hole burning (the spectrum is displaced on the extinction scale by 1.0 unit); (b) after hole burning. (c) Enlarged representation of the hole spectrum. The vibronic satellite holes are labeled with their distances from the hole resonant with the laser line $\left(\mathrm{cm}^{-1}\right)$.

ometries for the ground state and the lowest electronically excited state. The vibrational sidebands are located 700,1260 (shoulder) and $1630 \mathrm{~cm}^{-1}$ from the origin band. Additional very weak sidebands are observed about 2320,2780 and $3020 \mathrm{~cm}^{-1}$ above the origin. These may be ascribed to combinations of the bands at $1630+700 \mathrm{~cm}^{-1}, 1630+1260 \mathrm{~cm}^{-1}$ and $2 \times 1630 \mathrm{~cm}^{-1}$, respectively. However, a more detailed analysis (see below) will show that these values must be looked at with caution.

Irradiation of the sample with the $632.8 \mathrm{~nm}$ light $\left(15803 \mathrm{~cm}^{-1}\right)$ of a $\mathrm{He} / \mathrm{Ne}$ laser results in the appearance of a spectral hole resonant to the laser line
(Figs. $2 \mathrm{~b}$ and $2 \mathrm{c}$ ). This hole is accompanied by several sideholes in the region of the electronic origin band (Fig. $2 \mathrm{c}$ and Table 1 ). The energy differences between these sideholes and the resonant hole reflect excited state vibrational frequencies of modes coupling to the electronic transition [4-7].

The appearance of more than one sidehole after irradiation into a broad vibrational band is due to the spectral overlap of the inhomogeneous absorption bands of several vibronic transitions accompanying the same electronic excitation. These could not be resolved in earlier absorption studies on silicon phthalocyanines. In the hole-burning experiments irradiation into the overlapping bands excites several spectral subensembles, one for each vibronic transition. The corresponding holes in the region of the electronic origin show that at least seven different vibrations are 'hidden' in the first vibrational absorption band. An assignment of vibrations coupling to an electronic transition based on broad-band spectra is not reliable for these large molecules. In particular, no mode with a frequency corresponding to $700 \mathrm{~cm}^{-1}$ (as deduced from the broad-band spectra, see above) can be detected.

A comparison of the excited state vibrational frequencies of the SiPc monomer with modes found for other phthalocyanines is interesting. Many vibrations coupling to the electronic transition between the lowest excited states and the ground state could be resolved in emission and excitation spectra of metal phthalocyanines in Shpol'skii matrices [24]. In Table 1 the energies of the vibrations detected in the reported hole-burning experiments are compared to modes of different metal phthalocyanines in the corresponding energy region. For all modes found in the hole-burning experiments analogous vibrations occur for other phthalocyanine compounds. Most of the listed frequencies show a slight dependence on the central ion. This may be expected for modes in the inspected frequency range since they may interfere with the motions of the central ion.

A detailed characterization of the hole-burning mechanism is not attempted in this contribution. For short times (about several tens of minutes) after the irradiation process an increase of the broad-band absorption in the origin region of the $Q$ band of $\mathrm{Hex}_{3} \mathrm{SiO}(\mathrm{SiPc}) \mathrm{OSiHex}_{3}$ could be observed. This increase of extinction faded within approximately one 
Table 1

Comparison of the excited state vibrational frequencies of $\mathrm{Hex}_{3} \mathrm{SiO}(\mathrm{SiPc}) \mathrm{OSiHex}_{3}$ in an Ar matrix to corresponding vibrational frequencies obtained from emission and excitation spectra of different metal phthalocyanines (MPcs) $\left(\mathrm{cm}^{-1}\right.$ )

\begin{tabular}{|c|c|c|c|c|c|c|}
\hline \multirow{2}{*}{$\begin{array}{l}\text { Hole burning on } \\
\text { SiPc monomer " }\end{array}$} & \multicolumn{2}{|l|}{$\mathrm{MgPc}^{\mathrm{b}}$} & \multicolumn{2}{|l|}{$\mathrm{ZnPc}^{\mathrm{b}}$} & \multicolumn{2}{|l|}{ ClAlPc $^{b}$} \\
\hline & emission & excitation & emission & excitation & emission & excitation \\
\hline 502 & & 479 & 482 & 477 & & \\
\hline 557 & & 564 & & 568 & & \\
\hline 588 & & 580 & 592 & 587 & 572 & \\
\hline 680 & 679 & 678 & 681 & 673 & 685 & 679 \\
\hline 754 & 746 & 741 & 750 & 742 & 743 & 766 \\
\hline 782 & & & & & & 796 \\
\hline 832 & 827 & 835 & 836 & 832 & & 830 \\
\hline
\end{tabular}

"This work, excited state frequencies.

bs far as detected in Ref. [24].

hour but the holes remained unchanged. The holeburning process in the Ar matrix may occur by a photo-rearrangement of the axial trihexyl siloxy groups. A similar argument was used to explain the photoreaction of axially substituted magnesium porphyrins $[12,13]$.

\subsection{SiPc dimer}

The Q-band absorption of the dimeric compound $\mathrm{Hex}_{3} \mathrm{SiO}$ ( $\mathrm{SiPc}-\mathrm{O}-\mathrm{SiPc}$ ) OSiHex 3 in an Ar matrix shows a similar dependence on the environment as discussed for the monomer $\mathrm{Hex}_{3} \mathrm{SiO}$ ( $\mathrm{SiPc}$ ) OSiHex ${ }_{3}$. The origin band of the upper exciton state appears at $16040 \mathrm{~cm}^{-1}$ (Fig. 3a) and is blue-shifted by about $150 \mathrm{~cm}^{-1}$ compared to the absorption in tetrahydrofuran or cyclohexane $[15,23]$. The origin of the forbidden transition to the lower exciton state cannot be identified with comparable clarity. The low-energy onset of the broad absorption in an Ar matrix is found at approximately $13000 \mathrm{~cm}^{-1}$. This gives an estimate for the energy position of the origin in the absorption of the lower exciton state. Hence, a splitting of the lowest excited singlet state due to exciton coupling in $\mathrm{Hex}_{3} \mathrm{SiO}$ (SiPc-O-SiPc)OSiHex ${ }_{3}$ of at least 3000 $\mathrm{cm}^{-1}$ is estimated.

Due to the uncertain position of the origin band of the lower exciton state the discussion of the vibrational sidebands will focus on the upper exciton state. Two vibrational sidebands occur with energy separations of 690 and $1560 \mathrm{~cm}^{-1}$ from its electronic origin. These correspond to the vibrational satellites of

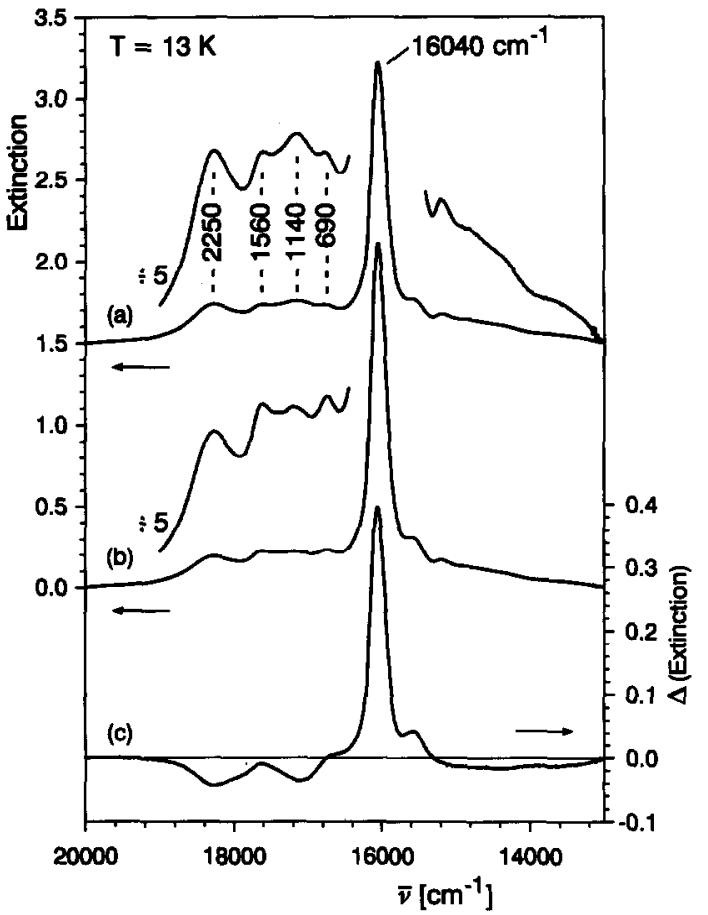

Fig. 3. Absorption spectra of the dimer $\mathrm{Hex}_{3} \mathrm{SiO}(\mathrm{SiPc}-\mathrm{O}-$ $\mathrm{SiPc}) \mathrm{OSiHex}_{3}$ in an Ar matrix at $T=13 \mathrm{~K}$, (a) before irradiation (the spectra are displaced on the extinction scale by 1.5 units); (b) after irradiation. (c) Difference spectrum of the absorption after irradiation minus the absorption before irradiation. The vibrational sidebands are marked with their distances to the electronic origin of the upper exciton state $\left(\mathrm{cm}^{-1}\right)$. The arrows indicate the scale which applies to the corresponding spectrum. 
the monomer $\mathrm{Hex}_{3} \mathrm{SiO}(\mathrm{SiPc}) \mathrm{OSiHex}_{3}$ at 700 and $1630 \mathrm{~cm}^{-1}$, respectively. In addition, two further bands with separations of 1140 and $2250 \mathrm{~cm}^{-1}$ from the origin can be found. In the absorption of the monomer no corresponding bands with comparable intensities are observed. Thus, the interaction between the two SiPc units should be the cause of the appearance of these two vibrational sidebands.

Irradiation of $\mathrm{Hex}_{3} \mathrm{SiO}\left(\mathrm{SiPc}-\mathrm{O}-\mathrm{SiPc}\right.$ )OSiHex $\mathrm{O}_{3}$ in an Ar matrix does not lead to the appearance of narrow holes. Instead, significant changes of the broadband absorption of the upper exciton state are observed (Fig. 3b). These changes (Fig. 3c) cannot be fit to Lorentzian profiles with satisfactory accuracy. The bands are better described by Gaussian curves, pointing to substantial inhomogeneities. For the absorption of the lower exciton state the extinction changes - although present - are not as clear. Moreover, due to the uncertain position of the origin band of the lower exciton state an analysis of the changes in the low-energy region of the absorption will not be attempted here.

The observed extinction changes may be explained by a photoreorientation of the entire molecule. (Perhaps the hexyl groups of the axial substituents are too flexible to prevent the heavy dimer molecules from rotating.) After irradiation of the samples into the inplane polarized origin absorption of the upper exciton state $\left(\lambda_{\text {exc }}=632.8 \mathrm{~nm}\right.$ corresponding to 15803 $\mathrm{cm}^{-1}$ ) the molecules tend to turn their in-plane absorption dipoles out of the direction of the electric field vector of the exciting light. Thus, for a polarization of the light for the absorption measurements perpendicular to the polarization of the laser light (Section 2) an increase in the in-plane polarized extinction and a decrease in an out-of-plane polarized extinction is expected.

In the difference spectrum shown in Fig. $3 \mathrm{c}$ it can be seen that the strong in-plane polarized origin band of the upper exciton states shows an increase in extinction after irradiation. A satisfactory fit of the difference spectrum can only be obtained if two positive bands for the vibrational sidebands at 690 and 1560 $\mathrm{cm}^{-1}$ are included. These sidebands correspond to the ones already present in the monomer. Thus, all bands which can be identified for the monomer as well as for the dimer show the same behavior upon irradiation. However, it is interesting that the vibrational sidebands at 1140 and $2250 \mathrm{~cm}^{-1}$, which are not present in the monomer absorption, show a marked decrease in their extinction (Figs. 3b and 3c). Moreover, in the difference spectrum (Fig. 3c) a third negative band with an energy separation from the origin of about $1800 \mathrm{~cm}^{-1}$ can be detected as a shoulder. Thus, these additional bands must be out-ofplane polarized - in contrast to the bands already present in the monomer. A control experiment irradiating the sample in an angle of $90^{\circ}$ to the beam path for the absorption measurements at the position of the band at $2250 \mathrm{~cm}^{-1}$ with light polarized in the plane of incidence of the spectrometer revealed the same extinction changes as shown in Fig. $3 \mathrm{c}$ with opposite signs thus confirming the presented results.

Due to the strong transition moment of the lowest $\pi-\pi^{*}$ excitations in the monomeric SiPcs only in-plane transitions are expected in the case of a pure excitontype coupling $[15,20]$. Thus, only a different interaction mechanism can be responsible for out-of-plane absorptions. Since the transition dipole for out-ofplane absorptions points from one phthalocyanine unit to the other (and vice versa), it is most probable that charge-resonance interactions contribute to the coupling between the two SiPc units. A similar interaction has been invoked to explain the coupling of the corresponding triplet states of dimeric SiPcs [22].

However, there should be a substantial difference between the relative importance of the exciton versus charge-resonance couplings for the singlet and the triplet states. The exciton coupling is proportional to the square of the corresponding transition moment (see e.g. Ref. [15]). Thus, the exciton coupling should be pronounced for the singlet states but it should vanish for the triplet states. Therefore, the charge-resonance contributions should dominate the interaction of the triplet states whereas for the singlet states the exciton interactions should be the main coupling process which can be concluded from the large exciton splitting of at least $3000 \mathrm{~cm}^{-1}$ combined with the forbidden transition to the lower exciton state.

\section{Conclusion}

The feasibility of spectral hole burning in silicon phthalocyanines isolated in an Ar matrix has been 
demonstrated. The mechanism for the appearance of spectral holes is as yet not fully clarified. A photorearrangement of the axial ligands may be responsible for the observed spectral changes. The appearance of well-resolved spectral holes in the absorption of the monomer $\mathrm{Hex}_{3} \mathrm{SiO}(\mathrm{SiPc}) \mathrm{OSiHex}_{3}$ yields valuable information on the vibrational structure in the Q-band region. For the dimer $\mathrm{Hex}_{3} \mathrm{SiO}$ ( $\mathrm{SiPc}-\mathrm{O}-$ $\mathrm{SiPc}) \mathrm{OSiHex}_{3}$ further investigations are in progress to obtain a similar resolution in the spectra. Nevertheless, already the changes in the polarized broadband absorption point to charge-resonance contributions to the excitonic interaction of the two SiPc units.

\section{Acknowledgement}

Financial support from the Belgian Government (Ministerie van Wetenschapsbeleid) and the Belgian National Science Foundation (NFWO) is gratefully acknowledged by the Leuven group. DB thanks the Onderzoeksraad KU Leuven for a research grant. The synthetic part was supported by a Grant-in-Aid for Scientific Research from the Japanese Government (MESC).

\section{References}

[1] R.M. Macfarlane, R.M. Shelby, J. Luminescence 36 (1987) 179.

[2] S. Völker, Ann. Rev. Phys. Chem. 40 (1989) 499.
[3] W.E. Moerner, ed., Topics in current physics, vol. 44. Persistent spectral hole-burning: science and applications (Springer, Berlin, 1988).

[4] B.M. Kharlamov, L.A. Bykovskaya, and R.I. Personov, Chem. Phys. Letters 50 (1977) 407.

[5] J. Friedrich and D. Haarer, J. Chem. Phys. 79 (1983) 1612

[6] J. Friedrich and D. Haarer, Angew. Chem. 96 (1984) 96.

[7] B. Dick, Chem. Phys. 136 (1989) 41 3, 429.

[8] S. Völker, J.H. van der Waals, Mol. Phys. 32 (1976) 1703.

[9] A.A. Gorokhovski, P.K. Kaarli and L.A. Rebane, JETP Letters 20 (1974) 216.

[10] R.M. Shelby and R.M. Macfarlane, Chem. Phys. Letters 64 (1979) 545.

[11] J. Hála, M. Vácha, J. Dian, F. Adamec, M. Ambroz and R. Tesar, Czech. J. Phys. 41 (1991) 373.

[12] R.J. Platenkamp, Mol. Phys. 45 (1982) 113.

[13] A.I.M. Dicker, L.W. Johnson, S. Völker and J.H. van der Waals, Chem. Phys. Letters 100 (1983) 8.

[14] M. Ehrl, F.W. Deeg, C. Bräuchle, O. Franke, A. Sobbi, G. Schulz-Ekloff and D. Wöhrle, J. Phys. Chem. 98 (1994) 47.

[15] N.S. Hush and I.S. Woolsey, Mol. Phys. 21 (1971) 465.

[16] H. Konami, M. Hatano and A. Tajiri, Chem. Phys. Letters $166(1990) 605$.

[17] E. Ciliberto, K.A. Doris, W.J. Pietro, G.M. Reisner, D.E. Ellis, I. Fragalà, F.H. Herbstein, M.A. Ratner and T.J. Marks, J. Am. Chem. Soc. 106 (1984) 7748.

[18] M. Moussavi, A. De Cian, J. Fischer and R. Weiss, Inorg. Chem. 27 (1988) 1287.

[19] B.L. Wheeler, G. Nagasubramanian, A.J. Bard, L.A. Schlechtman, D.R. Dininny and M.E. Kenney, J. Am. Chem. Soc. 106 (1984) 7404.

[20] R.L. Fulton, M. Gouterman, J. Chem. Phys. 41 (1964) 2280.

[21] P. Petelenz and M.Z. Zgierski, Mol. Phys. 25 (1973) 237.

[22] R. Miyamoto, S. Yamauchi, N. Kobayashi, T. Osa, Y. Ohba and M. Iwaizumi, Coord. Chem. Rev., in press.

[23] A.R. Kane, J.F. Sullivan, D.H. Kenny and M.E. Kenney, Inorg. Chem. 9 (1970) 1445.

[24] T.-H. Huang, K.E. Rieckhoff and E.M. Voigt, J. Chem. Phys. 77 (1982) 3424. 